\title{
Immunohistochemical Expression of P53 and Cyclin D1 Proteins in Follicular Thyroid Tumors: Evaluation of Their Significance
}

\author{
Ahmed H. Abdel-Rahman El-Rashidy ${ }^{1}$ - Samir Abdel Salam A. Ibraheim ${ }^{2}-$ \\ Amer Yehia Mohamed ${ }^{2}-$ Mohammed Hassan Fahmy ${ }^{3}$ - Shehata $\mathrm{AM}^{4}$ \\ From Departments of Pathology ${ }^{1}$ and General Surgery ${ }^{2,3,4}$, Al-Azhar(Assiut), Cairo \& Al- \\ Menya Universities
}

\begin{abstract}
The sequential mutational events which may underlie the tumorigenesis of thyroid neoplasia are gradually becoming apparent. To clarify the role of P53 and cyclin D1 in the oncogenesis and tumor progression of thyroid neoplasms, we examined the immunoreactivity of these proteins in one hundred and thirty (130) thyroid tumors originating from the follicular epithelium using immunohistochemistry. The tumors were divided into two groups group I: included thirty five (35) follicular adenomas and group II: included ninety five (95) follicular carcinomas; 65 of which were well differentiated (WDC) while the remaining (30) were poorly differentiated (PDC). P53 positivity was more frequent in group II $(20 / 95 ; 21.1 \%)$ than in group I $(6 / 35 ; 17.1 \%)$ and in PDC $(11 / 30 ; 26.6 \%)$ than WDC $(9 / 65 ; 13.8 \%)$. On the other hand, cyclin D1 positivity was frequent in WDC $(21 / 65 ; 32.3 \%)$ and rarely seen in adenoma group $(2 / 35$; $5.7 \%)$. Co - positivity for P53 and cyclin D1 proteins was more observed in PDC (5/30; 16.7\%) than in WDC $(3 / 65 ; 4.6 \%)$.

The study suggested that cyclin DI may be involved in the thyroid oncogenesis and concluded that both proteins may be incriminated in the progression of follicular thyroid neoplasms. Moreover, we found that age at the time of diagnosis, the histologic differentiation, necrosis in primary tumor, extrathyroidal invasion and the presence of distant metastases are important prognostic and risk factors. In addition, our study revealed that the detection of P53, but not cyclin D1, in primary follicular carcinomas is a significant independent prognostic indicator which, together with the above mentioned important prognostic factors, may be of value in the theraputic planning of these tumors.
\end{abstract}

Key words: Thyroid gland - Follicular tumors - Immunohistochemistry- P53 - Cyclin D1- prognosis.

\section{Introduction}

Despite numerous strategies to develop new treatments, cancer is still one of the main causes of mortality in developed countries. Indentification of prognostic factors and development of scoring systems might permit a selective approach to therapy, thereby avoiding unnecessarily aggressive treatment for tumors that are likely to follow a low malignant potential course and in addition, avoiding inadequate therapy for those anticipated to display an aggressive behavior (Ceraline et al.,2003).

The genetic alterations leading to the neoplastic transformation of the thyroid follicular epithelium are not completely understood. However, the transformation is reported to involve the activation of oncogenes and the loss of functional tumor suppressor genes (Dobashi et al.,1994; Pilotti et al.,1994 \& Motoi et la.,2000).

P53 gene, a tumor suppressor gene, located on the shart arm of chromosome 17, encodes a nuclear phosphoprotein of P53 $\mathrm{KDa}$ that functions as a transcription factor by binding DNA and negatively regulates cell division by blocking the cell cycle in GI-S transition (Josef, 1999). P53 inactivation occurs by point mutation and allelic loss or other mechanisms and has proved to be an important step for carcinogenesis (Jensen and Page,1993). The protein of mutated/inactivated P53 accumulates in the cell at the level detectable by immunohistochemistry, whereas wild type P53 normally does not. For this reason, P53 immunochemistry represents a possibility to get inside into the process of neoplastic 
transformation (Kataki et al., 2003). Cells with no or mutated P53 fail to block growth following DNA damage leading to instability and increasing numbers of transformed cells (Marx, 1993). Mutant P53 also appears to inhibit apoptosis ( Oltaviz and Korsmeyer, 1994).

Moreover, it has been suggested that P53 plays a role in the process of cell differentiation and that P53 alterations may therefore interfere with cell commitment towards differentiation (Battista et al ., 1995; Fagin et al.,1996 and Soddu et al.,1996).

Cyclins; cell cycle regulators, exert their effects by governing the cell cycle progression at various check points, and alterations in their expression might also contribute to tumorigenesis (Plamero and Peters, 1996). Among these cyclins, cyclin D1 is almost certainly involved in tumorigenesis. Isolation of cyclin D1 resulted from a screening for candidate oncogenes to promote the formation of parathyroid tumors (Motokura et al.,1991) and centrocyte B-cell lymphoma (Rosenberg et al.,1991). Overexpression of cyclin D1 has been reported in several types of human cancers (Nishida et al.,1994; Zhu et al.,1998 \& Watanabe et al.,1999). Cyclin D1 acts by complexing with cycledependent kinase edk 4 or edk 6 at late G1 of the cell cycle (Plamero and Peters, 1996). The activity of the cyclin D1/edk complex can be regulated by several inhibitory mechanisms, one of which is the pathway mediated by the P53 abnormalities observed in a variety of cancers including thyroid carcinoma (Dobashi et al.,1994; Pilotti et al.,1994 \& Goto et al.,2001).

This study elucidates the role of P53 and cyclin D1 proteins in the tumorigenesis and tumor progression of the thyroid neoplasms originating from the follicular epithelium. Moreover, it investigates the prognostic significance of these proteins in thyroid follicular carcinomas.

\section{Materials and methods}

This retrospective study was carried out on a sum of one hundred and thirty (130) primary follicular thyroid tumors obtained from patients undergoing lobect- omy $(32 ; 24.6 \%)$, subtotal thyroidectomy $(34 ; 26.2 \%)$ or total thyroidectomy (64; 49.2\%) at Al-Azhar University \& Kasr ElAini Hospitals during the period from March 1, 2003 to February 28, 2006. Adjuvant therapy was received in the form of radio-iodine in 29 (30.5\%) or external radiotherapy in 13 cases (13.8\%).

The cases, were divided into two groups (Table 1); group 1: included thirty five (35) follicular adenomas and group II: included ninety five (95) follicular carcinomas subdivided histologically into two grades; well differentiated (WDC; 65 cases) and poorly differentiated (PDC; 30 cases). Normal thyroid tissue adjacent to the primary lesions were used as controls.

The follow-up data as well as the clinicopathologic variables including age at the time of diagnosis, sex, distant metastases, histologic grade, tumor size, extrathyroidal growth (PT4), regional lymph node metastasis, tumor necrosis were obtained from the patients' files. The mean follow-up period was 40.2 months (range, 21 to 57 months). Twenty two patients $(23.2 \%)$ died of thyroid carcinomas while five patients $(5.3 \%)$ died of other causes. The average survival time was 48.2months (range, 11 to 60 months).

\section{Histopathological and immunohisto- chemical procedures: \\ Formalin-fixed, paraffin-embedded} blocks were sectioned at $5 \mu \mathrm{m}$ thickness. Three sections per each case were prepared; one was stained with hematoxylin and eosin for routine histopathologic study while the other two sections were used for immunohistochemical study as follows: Sections on poly-L-lysine coated glass slides were deparaffinized in xylene and rehydrated using ethanol gradients, then pretreated three times in a microwave oven for 5 minutes in citrate solution (BiogenexNeufahrn, Germany). Endogenous peroxidase activity was blocked by emmersion in $3 \%$ hydrogen methanol for 20 minutes. Washing three times with cold $0.01 \mathrm{M}$ phosphate buffered saline (PBS), then blocking with $10 \%$ normal rabbit serum followed by incubation for 12 hours at $4^{\circ} \mathrm{C}$ with mouse monoclonal antibodies against human cyclin D1 protein (5D4, Medical Biological Laboratories Ltd; Nagoya, 


\section{Ahmed H. Abdel et al}

Japan) and against human P53 (Do7, Novocastra Laboratories Ltd., Newcastle upon Tyne, U.K.). Incubation with biotylated rabbit antimouse $\mathrm{IgG}$ followed by addition of the streptavidin-biotin peroxidase reagent, (Histofine Kit, Nichirei Corp., Tokyo, Japan). Finally, the chromogen, diaminobenzidine and $3 \%$ hydrogen peroxidase were applied to the sections.

\section{Assessment of immunoreactivity}

Positive reaction for cyclin D1 appeared as brownish nuclear staining while P53 immunopositivity was observed as brownish nuclear and occasionally cytoplasmic staining. The percentage of tumor cells in each section exhibiting positive immunostaining was estimated by counting, at least, 500 tumor cell nuclei in the most five representative areas of the primary tumor. An index between the number of P53-or cyclin D1-positive cells and the total number of counted cells was calculated. The evaluation of the immunocytochemical results of P53 and cyclin D1 expression was performed taking into account the density and the intensity of reactive cells and quantitated in the following frequency score: $\mathrm{O}$; negative $(0-$ $5 \%), 1+(6-10 \%), 2+(11-\leq 50 \%)$ or $3+$ (> 50\% reactive cells). The intensity score was assigned as weak (1+), moderate $(2+)$ or strong $(3+)$.

\section{Statistical analysis}

It was performed using the chi square or Fisher's exact probability test. For multivariate analysis, Cox proportional model was applied. Variables that showed significant influence on survival in univariate analysis were included in the multivariate one. The hazard ratio corresponding to each factor in the study was determined. A probability levels of $\leq$ 0.05 was used to indicate a statistical significance.

\section{Results}

\section{Clinicomorphological data:}

The female/male ratio was 4.8:1 (No; 29:6) in group I and 4: 1 (No; 76: 19) in group II $(\mathrm{P}=0.163)$. The median age was 32.8 years in groups $\mathrm{I}(\mathrm{SD}=4.7$; range, 21 to 52 years) and 48.7 years $(\mathrm{SD}=15.5$; range, 36 to 79 years) in group II $(\mathrm{P}=$ $0.013)$. The median tumor size was $3.3 \mathrm{~cm}$ in group $\mathrm{I}(\mathrm{SD}=1.4$; range, 1.5 to $3.6 \mathrm{~cm})$ and $3.1 \mathrm{~cm}$ in group II ( $\mathrm{SD}=1.9$; range, 1.4 to $5.2 \mathrm{~cm} ; \quad \mathrm{P}=0.121)$. Histologic examination of the adenoma group (Fig. 1) showed discrete fibrous encapsulation together with proliferated small and /or large sized follicles containing little or no colloid \& lined by bland cubical epithelium. Moreover, the tumors in group II were classified into well differentiated composed of well-defined and variable sized follicles (Fig.2) and poorly differentiated carcinomas showing solid-insular and/or trabecular patterns (Fig. 3).

\section{Immunohistochemistry:}

P53 staining: Immunoreactivity for P53 showed insignificant difference between both groups (Table 2) being found in $17.1 \%(6 / 35)$ of group I (Fig. 4) and in $21.1 \%(20 / 95)$ of group II cases.

Also, the frequency and intensity scores were relatively higher in group II compared to the group I as the adenoma group often displayed low P53 scores while most of the carcinoma group showed high P53 scores. Among the group II, 9 out 65 (13.8\%) WDC (Fig. 5) and 11 out of 30 (26.6\%) PDC were P53-positive. Moreover, 4 positive out of 63 cases $(3.6 \%)$ were localized (No Mo); two of which were $\leq$ $35 \mathrm{~mm}\left(\mathrm{~T}_{1}+\mathrm{T}_{2}\right)$ and the remainings were $>$ $35 \mathrm{~mm}\left(\mathrm{~T}_{3}\right)$, while 16 positive out of 32 cases $(50 \%)$ were metastatic (N1 \&/or M1). A significant correlation was noticed between P53 positivity and either of the histologic differentiation degree or $\mathrm{T}$-stage or the clinical stage. The normal control thyroid tissue was constantly negative for P53.

Cyclin D1 staining: Immunoreactivity for cyclin D1 was more observed in group II $(33.7 \%$; 32/95) than in group 1 $(5.7 \% ; 2 / 35)$ with a significant difference between both group (Table 3). Also, the frequency and intensity scores were relatively more predominant in carcinoma than in adenoma group (Fig. 6). Within the group II, 21 out of $65(32.3 \%)$ were WDC (Fig. 7) while 11 out of $30(36.7 \%)$ were PDC. Moreover, 20 positive out of 63 $(31.7 \%)$ were localized (NoMo), 16 of 
which were $\leq 35 \mathrm{~mm}\left(\mathrm{~T}_{1}+\mathrm{T}_{2}\right)$ and the remainings were $>35 \mathrm{~mm}\left(\mathrm{~T}_{3}\right)$, while 12 positive out of 32 cases (37.5\%) were metastatic (N1 \& /or M1). There was insignificant correlation between cyclin D1 and each of the histologic differentiation degree, T-stage and the clinical stage. The normal control thyroid tissue was also negative for cyclin D1.

Relationship between P53 and cyclin D1 immunoreactivity (Table 4): The adenoma group showed the following profile: Each of P53 (+) /cyclin D1 (+) and P53 (-)/cyclin D1 (+) found in 2.9\% (1/35), P53 (+)/ cyclin D1 (-) in $14.3 \%(5 / 35)$ and P53 (-) / cyclin D1 (-) in 80\% (28/35). On the other hand, the carcinoma group displayed the following profile: P53 (+)/ cyclin D1 (+) in 13 cases $(13.7 \%)$; seven of which $(10.8 \%)$ were WDC while the remaining six (20\%) were PDC, P53 (-) /cyclin D1 (+) in 19 cases including fourteen $(21.5 \%)$ WDC \& five $(16.7 \%)$ PDC, P53 (+) /cyclin D1 (-) in 7 cases (7.4\%), of which two $(3.1 \%)$ were WDC while five (16.7\%) were PDC, and P53 (-) / cyclin D1 (-) in 56 cases involving fourty two $(64.6 \%)$ WDC \& fourteen $(46.7 \%)$ PDC. A significant difference $(\mathrm{P}<0.05)$ was observed between both groups regarding P53 (+) and /or cyclin D1 (+) phenotypes. In addition, among the group II cases, a significant difference was found between both histologic differentiation degrees regarding P53 (+) /cyclin D1 (+) and P53 (+) /cyclin D1 (-) phenotypes. However, P53 (-)/cyclin D1 (+) phenotype insignificantly $(\mathrm{P}=0.141)$ displayed between WDC \& PDC. On the contrary, absence of both molecules; P53 (-) /cyclin D1 (-), showed a significant difference between both groups and also between both degrees of differentiation being higher in adenoma group (80\%) and WDC (64.6\%) than in carcinoma group (58.9\%) and PDC $(46.7 \%)$ respectively. Pearson correlation analysis revealed that P53 and cyclin D1 were independently expressed $(P=0.261)$ as in P53 negative cases, cyclin D1 was more displayed in WDC than in PDC and FA while in cyclin negative cases, P53 was found in a higher percentage in PDC than in WDC and FA.

Univariate survival analysis (Table 5) showed that most of group II clinicopathologic variables including the age, tumor size, distant metastases, histologic differentiation, extrathyroidal invasion, tumor necrosis and P53 expression were significant $(\mathrm{P}<0.0001)$ prognostic indicators and significantly affect the causespecific survival. On contrast, sex, lymph nodal metastases and cyclin D1 expression were insignificant indicators and didn't significantly affect the survival.

Variables with significant influence on survival in univariate analysis were introduced in Cox multivariate analysis (Table 6) which included sixty five patients $(67.4 \%)$ for whom all informations were available. There were insignificant differences in relevant clinicomorphologic parameters between the group II cases included and not included $(30 / 95 ; 32.7 \%)$ in this analysis which revealed that all involved parameters except the tumor size, had a significant $(\mathrm{P}<0.0001)$ influence on the cause-specific survival.

Table (1): Histological distribution of cases $(n=130)$ :

\begin{tabular}{|c|c|}
\hline Diagnosis & No., of cases \\
\hline Group I: Follicular adenomas (FA) & 35 \\
Group II: Follicular carcinoma (FC): & 95 \\
Well differentiated (WDC) & 65 \\
Poorly differentiated (PDC) & 30 \\
\hline Total & $\mathbf{1 3 0}$ \\
\hline
\end{tabular}


Ahmed H. Abdel et al

Table (2): P53 positivity in all studied cases $(n=130)$ :

\begin{tabular}{|c|c|c|c|c|c|c|c|c|c|c|}
\hline \multirow{3}{*}{ Group } & \multicolumn{9}{|c|}{ P53 Positivity } & \multirow[b]{3}{*}{$P$ value } \\
\hline & \multirow[b]{2}{*}{ No } & \multirow[b]{2}{*}{$\%$} & \multicolumn{4}{|c|}{ FS } & \multicolumn{3}{|c|}{ IS } & \\
\hline & & & $\mathbf{0}$ & $1+$ & $2+$ & $3+$ & $\begin{array}{c}\mathrm{W} \\
(\mathbf{1 +})\end{array}$ & $\begin{array}{c}\mathrm{M} \\
(2+)\end{array}$ & $\begin{array}{c}\mathbf{S} \\
(3+)\end{array}$ & \\
\hline $\begin{array}{l}\text { Group I: Adenomas }(\mathrm{n}=35) \\
\text { Group II: Carcinomas }(\mathrm{n}=95) \text { : }\end{array}$ & $\begin{array}{l}6 \\
20\end{array}$ & $\begin{array}{l}17.1 \\
21.1\end{array}$ & $\begin{array}{l}2 \\
2\end{array}$ & $\begin{array}{l}3 \\
8\end{array}$ & $\begin{array}{l}1 \\
6\end{array}$ & $\begin{array}{l}0 \\
4\end{array}$ & $\begin{array}{l}4 \\
3\end{array}$ & $\begin{array}{l}2 \\
11\end{array}$ & $\begin{array}{l}0 \\
6\end{array}$ & $\mathrm{NS}^{\bullet}$ \\
\hline $\begin{array}{l}\text { Histologic degree of differentiation: } \\
\text { WDC }(n=65) \\
\text { PDC }(n=30)\end{array}$ & $\begin{array}{l}9 \\
11 \\
\end{array}$ & $\begin{array}{l}13.8 \\
26.6 \\
\end{array}$ & $\begin{array}{l}1 \\
1\end{array}$ & $\begin{array}{l}5 \\
3\end{array}$ & $\begin{array}{l}2 \\
4\end{array}$ & $\begin{array}{l}1 \\
3\end{array}$ & $\begin{array}{l}2 \\
4\end{array}$ & $\begin{array}{l}5 \\
6\end{array}$ & $\begin{array}{l}1 \\
2\end{array}$ & $<0.05$ \\
\hline $\begin{array}{l}\text { TMN stage: } \\
\text { T1+2 No Mo }(\mathrm{n}=53) \\
\text { T3 NoMo }(\mathrm{n}=10) \\
\mathrm{T} \text { any N1M0+T any N any M1 (n } \\
=32)\end{array}$ & $\begin{array}{l}2 \\
2 \\
16\end{array}$ & $\begin{array}{l}3.7 \\
20 \\
50\end{array}$ & $\begin{array}{l}0 \\
0 \\
2\end{array}$ & $\begin{array}{l}1 \\
0 \\
7\end{array}$ & $\begin{array}{l}1 \\
1 \\
4\end{array}$ & $\begin{array}{l}0 \\
1 \\
3\end{array}$ & $\begin{array}{l}1 \\
0 \\
5\end{array}$ & $\begin{array}{l}1 \\
1 \\
9\end{array}$ & $\begin{array}{l}0 \\
1 \\
2\end{array}$ & $<0.05$ \\
\hline $\begin{array}{l}\text { Clinical stage : } \\
\text { Localized; No M0 }(\mathrm{n}=63) \\
\text { Metastatic, N1 \&/or M1 }(\mathrm{n}=32)\end{array}$ & $\begin{array}{l}4 \\
16\end{array}$ & $\begin{array}{l}6.3 \\
50\end{array}$ & $\begin{array}{l}0 \\
2 \\
\end{array}$ & $\begin{array}{l}1 \\
7\end{array}$ & $\begin{array}{l}2 \\
4 \\
\end{array}$ & $\begin{array}{l}1 \\
3\end{array}$ & $\begin{array}{l}1 \\
5\end{array}$ & $\begin{array}{l}2 \\
9 \\
\end{array}$ & $\begin{array}{l}1 \\
2\end{array}$ & $<0.05$ \\
\hline Normal thyroid & 0 & 0 & 0 & 0 & 0 & 0 & 0 & 0 & 0 & \\
\hline $\begin{array}{l}F S=\text { Frequency score } \\
\text { W = Weak } \\
\bullet \text { NS = Not significant } \\
T_{1}<20 \mathrm{~mm}\end{array}$ & & $M=M o$ & rate; & & $\begin{array}{l}I S=I \\
S=S t\end{array}$ & $\begin{array}{l}\text { ensity } \\
\text { ong }\end{array}$ & core & & & \\
\hline
\end{tabular}

Table (3): Cyclin D1 positivity in all studied cases $(n=130)$ :

\begin{tabular}{|c|c|c|c|c|c|c|c|c|c|c|}
\hline \multirow{3}{*}{ Group } & \multicolumn{9}{|c|}{ Cyclin D1 Positivity } & \multirow{3}{*}{$P$ value } \\
\hline & \multirow[b]{2}{*}{ No } & \multirow[b]{2}{*}{$\%$} & \multicolumn{4}{|c|}{ FS } & \multicolumn{3}{|c|}{ IS } & \\
\hline & & & $\mathbf{0}$ & $1+$ & $2+$ & $3+$ & $\begin{array}{c}\mathbf{W} \\
(1+)\end{array}$ & $\begin{array}{c}\mathrm{M} \\
(2+)\end{array}$ & $\begin{array}{c}\mathbf{S} \\
(3+)\end{array}$ & \\
\hline $\begin{array}{l}\text { Group I: Adenomas }(\mathrm{n}=35) \\
\text { Group II: Carcinomas }(\mathrm{n}=95) \text { : }\end{array}$ & $\begin{array}{l}2 \\
32\end{array}$ & $\begin{array}{l}5.7 \\
33.7\end{array}$ & $\begin{array}{l}0 \\
4\end{array}$ & $\begin{array}{l}1 \\
6\end{array}$ & $\begin{array}{l}1 \\
10\end{array}$ & $\begin{array}{l}0 \\
12\end{array}$ & $\begin{array}{l}1 \\
9\end{array}$ & $\begin{array}{l}1 \\
13\end{array}$ & $\begin{array}{l}0 \\
10\end{array}$ & $<0.05$ \\
\hline $\begin{array}{l}\text { Histologic degree of differentiation: } \\
\text { WDC }(n=65) \\
\text { PDC }(n=30)\end{array}$ & $\begin{array}{l}21 \\
11\end{array}$ & $\begin{array}{l}32.3 \\
36.7\end{array}$ & $\begin{array}{l}3 \\
1\end{array}$ & $\begin{array}{l}5 \\
1\end{array}$ & $\begin{array}{l}6 \\
4\end{array}$ & $\begin{array}{l}7 \\
5\end{array}$ & $\begin{array}{l}6 \\
3\end{array}$ & $\begin{array}{l}9 \\
4\end{array}$ & $\begin{array}{l}6 \\
4\end{array}$ & NS • \\
\hline $\begin{array}{l}\text { TMN stage: } \\
\text { T1+2 No Mo }(\mathrm{n}=53) \\
\text { T3 NoMo }(\mathrm{n}=10) \\
\mathrm{T} \text { any N1M0+ T any N any M1 (n } \\
=32)\end{array}$ & $\begin{array}{l}16 \\
4 \\
12\end{array}$ & $\begin{array}{l}30.2 \\
40 \\
37.5\end{array}$ & $\begin{array}{l}2 \\
1 \\
1\end{array}$ & $\begin{array}{l}5 \\
0 \\
1\end{array}$ & $\begin{array}{l}3 \\
1 \\
6\end{array}$ & $\begin{array}{l}6 \\
2 \\
4\end{array}$ & $\begin{array}{l}3 \\
2 \\
4\end{array}$ & $\begin{array}{l}6 \\
2 \\
5\end{array}$ & $\begin{array}{l}7 \\
0 \\
3\end{array}$ & $\mathrm{NS} \bullet$ \\
\hline $\begin{array}{l}\text { Clinical stage: } \\
\text { Localized; No M0 (n =63) } \\
\text { Metastatic; N1 \& / or M1 (n = 32) }\end{array}$ & $\begin{array}{l}20 \\
12\end{array}$ & $\begin{array}{l}31.7 \\
37.5\end{array}$ & $\begin{array}{l}3 \\
1\end{array}$ & $\begin{array}{l}5 \\
1\end{array}$ & $\begin{array}{l}4 \\
6\end{array}$ & $\begin{array}{l}8 \\
4\end{array}$ & $\begin{array}{l}5 \\
4\end{array}$ & $\begin{array}{l}8 \\
5\end{array}$ & $\begin{array}{l}7 \\
3\end{array}$ & NS • \\
\hline Normal thyroid & 0 & 0 & 0 & 0 & 0 & 0 & 0 & 0 & 0 & \\
\hline $\begin{array}{l}F S=\text { Frequency score } \\
W=\text { Weak } \\
\bullet \text { NS = Not Significant } \\
T_{l}<20 \mathrm{~mm}\end{array}$ & & $M=M o$ & rate; & & $\begin{array}{l}I S=I \\
S=S t\end{array}$ & $\begin{array}{l}\text { ensity } \\
\text { ng }\end{array}$ & core & & & \\
\hline
\end{tabular}


Table (4): Relationship between P53 and cyclin D1 immunoreactivities in all studied cases $(n=130)$ :

\begin{tabular}{|c|c|c|c|c|c|c|c|c|}
\hline \multirow[t]{3}{*}{ Phenotype } & \multirow{2}{*}{\multicolumn{2}{|c|}{$\begin{array}{c}\text { Group I } \\
(\mathbf{n}=\mathbf{3 5})\end{array}$}} & \multicolumn{6}{|c|}{ Group II (n =95) } \\
\hline & & & & & WD & = 65) & & $=30)$ \\
\hline & No & $\%$ & No & $\%$ & No & $\%$ & No & $\%$ \\
\hline P53 (+) /cyclin D1 (+) & 1 & 2.9 & 13 & $13.7 *$ & 7 & 10.8 & 6 & $20^{*}$ \\
\hline P53 (-)/cyclin D1 (+) & 1 & 2.9 & 19 & $20 *$ & 14 & 21.5 & 5 & $16.7 \bullet$ \\
\hline P53 (+)/cyclin D1 (-) & 5 & 14.3 & 7 & $7.4 *$ & 2 & 3.1 & 5 & $16.7 *$ \\
\hline P53 (-)/cyclin D1 (-) & 28 & 80 & 56 & $58.9 *$ & 42 & 64.6 & 14 & $46.7 *$ \\
\hline
\end{tabular}

$W D C=$ Well differentiated carcinoma $; \quad P D C=$ Poorly differentiated carcinoma. $\%=$ Percentage of the number of each phenotype divided by the total number of corresponding group or subgroups cases

- Insignificant $P$ value (=0.141); $\quad$ *significant $P$ value $(\leq 0.05)$.

Table (5): Prognostic indicators in group II cases using univariate survival analysis $(\mathbf{n}=95)$ :

\begin{tabular}{|c|c|c|c|c|}
\hline \multirow[t]{2}{*}{ Clinicopathologic Indicators } & \multirow[t]{2}{*}{ Total No. } & \multicolumn{2}{|c|}{$\begin{array}{c}5 \text { year cause -specific॰• } \\
\text { survival }\end{array}$} & \multirow[t]{2}{*}{$P$ value * } \\
\hline & & No & $\%$ & \\
\hline $\begin{array}{l}\text { Age (years): } \\
\leq 45 \\
>45\end{array}$ & $\begin{array}{c}33(28) \mathrm{a} \\
62(37)\end{array}$ & $\begin{array}{l}25 \\
23\end{array}$ & $\begin{array}{l}89.3 \\
62.2 \\
\end{array}$ & $<0.0001$ \\
\hline $\begin{array}{l}\text { Sex: } \\
\text { Female } \\
\text { Male }\end{array}$ & $\begin{array}{l}76(53) \\
19(12)\end{array}$ & $\begin{array}{c}40 \\
9 \\
\end{array}$ & $\begin{array}{l}75.5 \\
75.0\end{array}$ & NS • \\
\hline $\begin{array}{l}\text { Tumor size }(\mathrm{mm}): \\
<35 \\
>35\end{array}$ & $\begin{array}{l}56(38) \\
39(27)\end{array}$ & $\begin{array}{l}35 \\
16\end{array}$ & $\begin{array}{l}92.2 \\
59.3\end{array}$ & $<0.0001$ \\
\hline $\begin{array}{l}\text { Lymph node metastases (PN): } \\
\text { Present } \\
\text { Absent }\end{array}$ & $\begin{array}{l}27(19) \\
68(46)\end{array}$ & $\begin{array}{c}15 \\
3 \\
\end{array}$ & $\begin{array}{l}78.9 \\
71.7\end{array}$ & NS • \\
\hline $\begin{array}{l}\text { Distant metastases }(\mathrm{M}): \\
\text { Present } \\
\text { Absent }\end{array}$ & $\begin{array}{l}32(9) \\
63(56)\end{array}$ & $\begin{array}{c}3 \\
49\end{array}$ & $\begin{array}{l}33.3 \\
87.5\end{array}$ & $<0.0001$ \\
\hline $\begin{array}{l}\text { Histologic degree of differentiation: } \\
\text { WDC } \\
\text { PDC }\end{array}$ & $\begin{array}{l}65(49) \\
30(16)\end{array}$ & $\begin{array}{c}42 \\
9\end{array}$ & $\begin{array}{l}85.7 \\
56.3\end{array}$ & $<0.0001$ \\
\hline $\begin{array}{l}\text { Extrathyroid invasion (PT4): } \\
\text { Present } \\
\text { Absent }\end{array}$ & $\begin{array}{l}43(24) \\
52(41)\end{array}$ & $\begin{array}{l}13 \\
37\end{array}$ & $\begin{array}{l}54.2 \\
90.2 \\
\end{array}$ & $<0.0001$ \\
\hline $\begin{array}{l}\text { Primary tumor necrosis: } \\
\text { Present } \\
\text { Absent }\end{array}$ & $\begin{array}{c}26(8) \\
69(57)\end{array}$ & $\begin{array}{c}3 \\
49 \\
\end{array}$ & $\begin{array}{l}37.5 \\
85.9\end{array}$ & $<0.0001$ \\
\hline $\begin{array}{l}\text { P53 expression: } \\
\text { Present } \\
\text { Absent }\end{array}$ & $\begin{array}{l}20(13) \\
75(52)\end{array}$ & $\begin{array}{c}9 \\
42 \\
\end{array}$ & $\begin{array}{l}69.2 \\
80.8 \\
\end{array}$ & $<0.0001$ \\
\hline $\begin{array}{l}\text { Cyclin D1 expression: } \\
\text { Present } \\
\text { Absent }\end{array}$ & $\begin{array}{l}42(30) \\
53(35)\end{array}$ & $\begin{array}{l}25 \\
22\end{array}$ & $\begin{array}{l}63.3 \\
62.9\end{array}$ & NS • \\
\hline
\end{tabular}

a: Number in parentheses denotes number of patients alive, and adequate for estimation of 5 year survival.

- Only deaths from thyroid carcinoma and considered events

- NS = Not Significant

$W D C=$ Well differentiated carcinomas. $\quad P D C=$ Poorly differentiated carcinomas.

* Log rank test for difference in survival between two groups. The test \& $P$ values are based on all patients and events for the whole follow-up period. 
Table (6): Effect of clinicopathologic variables on group II survival using multivariate analysis $(n=65)$ :

\begin{tabular}{|c|c|c|c|}
\hline Clinicopathologic Variables & HR** & 95\% CI*• & $P$ value \\
\hline Age $\leq 45$ versus $>45$ years & 1.590 & $1.205-3.673$ & $0.0005^{*}$ \\
\hline Tumor size $\leq 35$ versus $>35 \mathrm{~mm}$ & 0.473 & $0.942-2.827$ & $0.1260 \bullet$ \\
\hline Distant metastasis: (+) Vs (-) & 1.850 & $1.192-4.627$ & $0.0046^{*}$ \\
\hline WDC versus PDC & 3.182 & $1.921-6.813$ & $0.0003 *$ \\
\hline Primary tumor necrosis: (+) Vs (-) & 2.735 & $1.317-3.750$ & $0.0028 *$ \\
\hline Extrathyroid invasion : (+) Vs (-) & 2.511 & $1.172-5.651$ & $0.0005^{*}$ \\
\hline P53 expression: (+) Vs (-) & 2.216 & $1.526-4.341$ & $0.0035^{*}$ \\
\hline
\end{tabular}

** HR = Hazards ratio,

$* \bullet C I=$ Confidence intervals

$W D C=$ Well differentiated carcinoma $; P D C=$ Poorly differentiated carcinoma

- Insignificant P value;

* Significant $P$ value
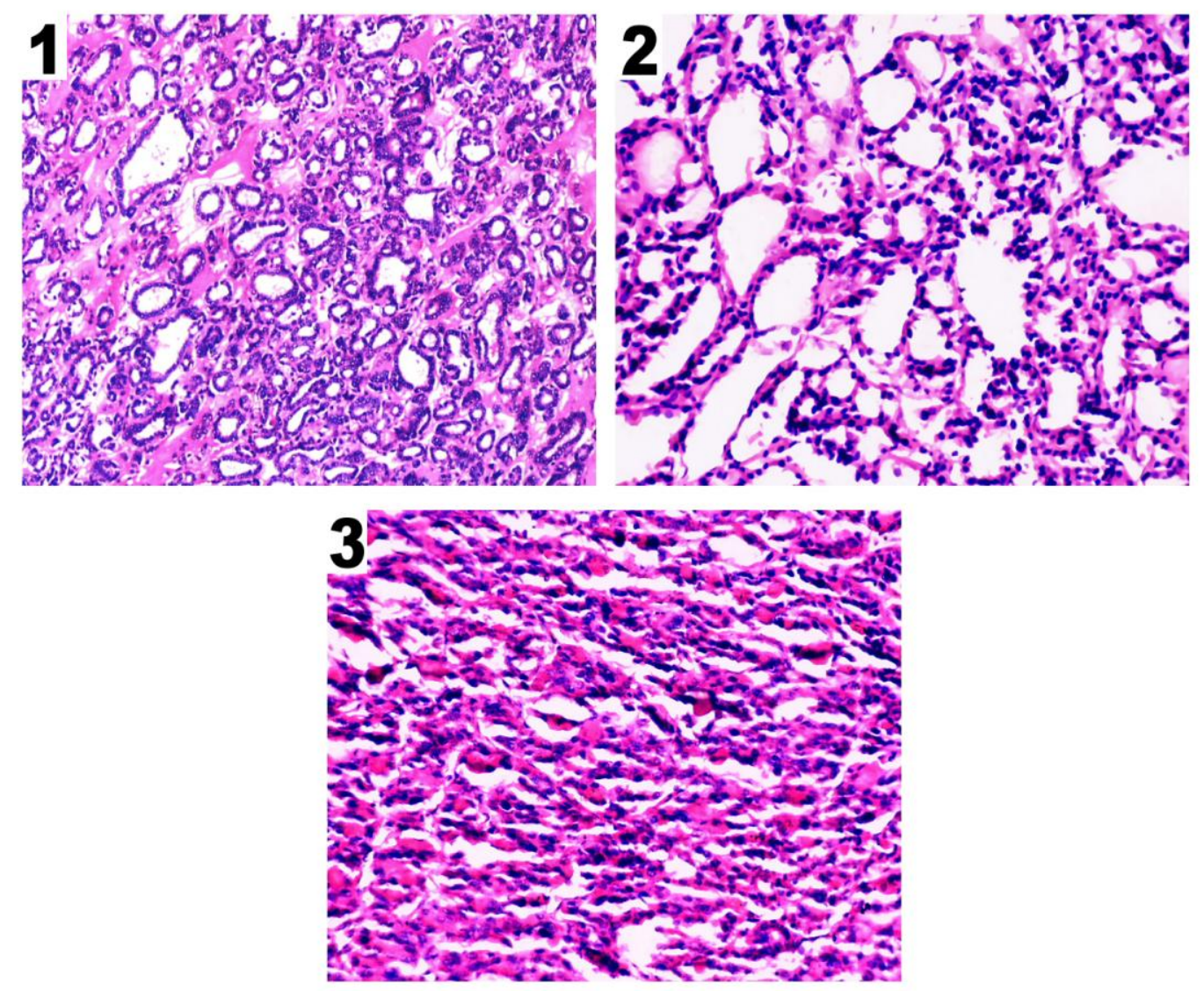

Figure - 1: Represents thyroid follicular adenoma (FA) showing small and large sized follicles lined by bland cells.

(H\&E X 100).

Figure - 2: Represents well differentiated follicular carcinoma (WDC) showing well defined and variable sized follicles lined by hyperchromatic malignant cells. $\quad$ (H\&E 200).

Figure - 3: Represents poorly differentiated follicular carcinoma (PDC) showing a trabecular pattern formed by hyperchromatic malignant cells.

(H\&E X 200$)$. 

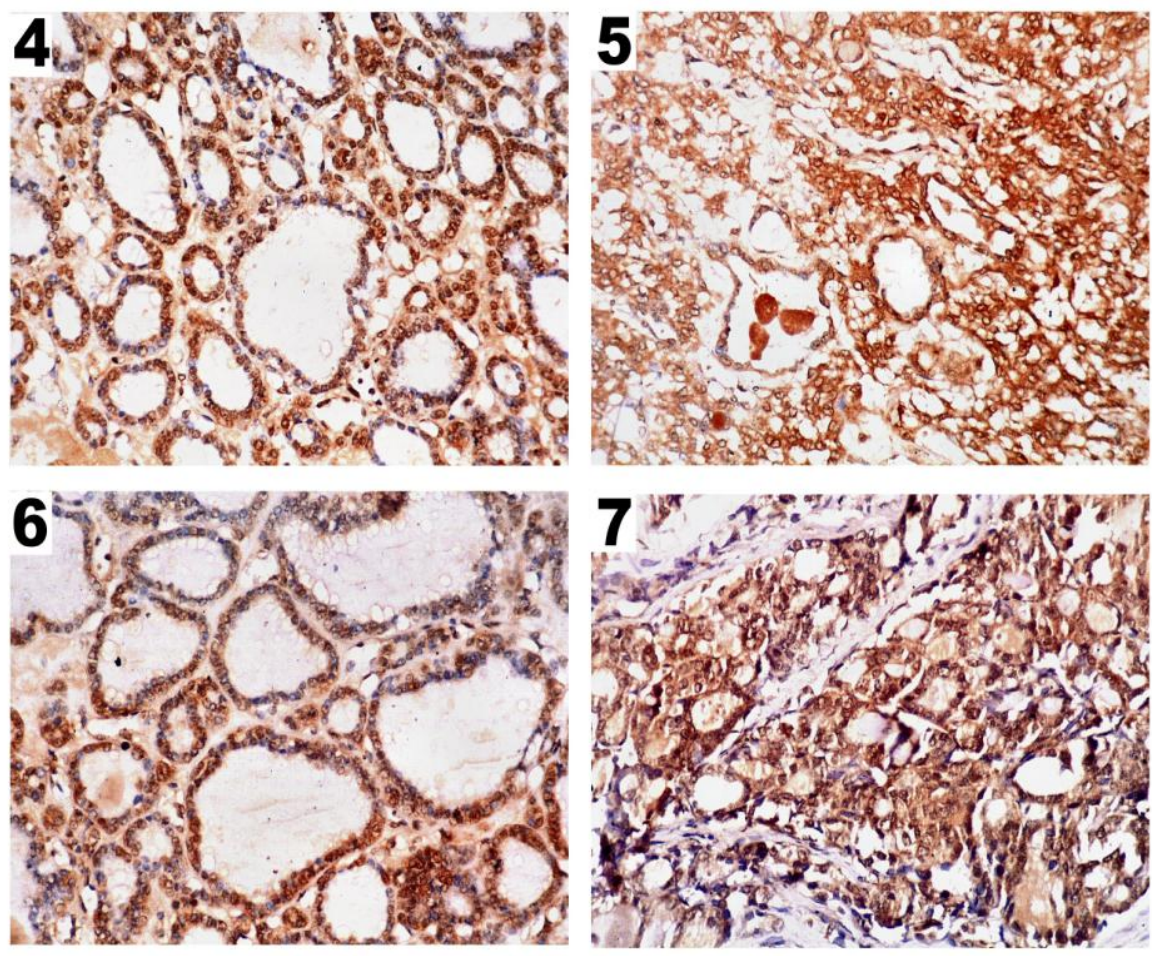

Figures 4 \& 5: Represent, respectively, FA moderately and WDC strongly positive for P53. Figures 6 \& 7: Represent, respectively, FA and WDC moderately positive for Cyclin D1.

\section{Discussion}

(Immunoperoxidase X200).

Thyroid tumorigenesis is characterized by progressive impairment of cell differentiation, resulting in loss of the highly specialized functions of the thyrocyte and acquisition of a more malignant behavior (Lo et al.,2005).

In the present study, P53 molecule was immunodetected in the nuclei and occasionally in the cytoplasm of tumor cells whileas cyclin D1 molecule was only immunolabelled in the tumor nuclei. These data coincided with those mentioned by $\mathrm{Ho}$ et al.(1996); Wang et al.(2000)\& Goto et al.(2001) who stated that the cytoplasmic cyclin D1 immunodetection in the thyroid neoplasms, even when found, had not being considered a significant true overexpression.

P53 immunopositivity was insignificantly more in group II than in group I while cyclin D1 expression was significantly greater in group II than in group I $(\mathrm{P}<$ $0.05)$. In addition, both molecules displayed higher frequency and intensity scores in carcinoma than in adenoma group. Neither of these molecules could be detected in the normal thyroid tissue. These results indicated that cyclin D1 and, to a lesser extent, P 53 overexp-ression may contribute to the malignant transformation of thyroid follicular epithel-ium and may be involved in the progression of thyroid neoplasms. Our findings agreed with those reported by Okayasu et al.(1998); Josef (1999)\&Goto et al.(2001) who discovered a disturbance of cell cycling which might be of importance to the eventual development of follicular epithelial cell tumors of thyroid. Moreover, Nasir et al.(2004) exhibited a strong P53 staining in $90 \%$ of follicular carcinomas and a weak staining in only $15 \%$ of follicular adenomas of the thyroid $(\mathrm{P}<0.0001)$ suggesting that this molecule may have a practical utility in differential diagnosis of both thyroid neoplasms in routine surgical pathology.

In addition, the study showed a significant correlation $(\mathrm{P}<0.05)$ between P53 positivity and either histologic differentiation, $\mathrm{T}$-or clinical stage of the group II cases; P53 - immunolabelled cells were more found in PDC than in WDC, in 
large than in small and in metastatic than in localized neoplasms, suggesting that P53 overexpression participated in the biological aggressiveness of follicular tumors particularly of histologically poorly differentiated subtype. In contrast, cyclin D1 positivity showed an insignificant correlation with either of the above mentioned parameters and was more frequent in WDC than PDC, in small than large and in localized than metastatic tumors. Also, Pearson correlation analysis revealed that both molecules were independently expressed in both groups. These results were similar to what were mentioned by Christian et al.(1998);Goto et al.(2001)\& Hod et al.(2005) who reported that P53 might be closely related to the tumor progression and development of PDC originated from WDC. This hypothesis is supported by the results of Wang and Harris (1996) who found that wild-type P53 has tumor suppressor functions such as cell-cycle arrest, DNA repair and induction of apoptosis, to prevent cell proliferation with damaged or altered DNA. Thus, loss of these functions of wild-type P53, caused by P53 abnormalities, might lead to the development of these thyroid neoplasms.

Combined analysis of P53 and cyclin D1 expression revealed a significant difference in the percentages of any phenotype between the two groups. Moreover, a significant difference in the percentages between group II cases that were either positive or negative for both molecules and their histologic differentiation; a higher percentage of P53 (+)/cyclin D1 (+) phenotype was seen in PDC while P53 ()/cyclin D1 (-) phenotype had a higher percentage in WDC. These findings were mimicking those reported by Lazzeresci et al.(1998); Wang et al. (1998) \&Goto et al. (2001) who suggested that the pathways through which P53 exerts its regulatory effect on the progression of the cell cycle are more frequently deregulated in cyclin D1-positive PDCs than in cyclin D1positive WDCs. Also, they stated that the deregulation of P53-mediated cell cycle regulation might play an important role in the development of PDCs from WDCs, especially in cases with cyclin D1 overexpression.
Our study displayed insignificant differences between both groups of follicular neoplasms regarding the sex and the tumor size. However, the mean age of carcinoma patients was significantly higher $(\mathrm{P}<0.05)$ than that of adenoma patients. Also, a statistical correlation between the possible prognostic factors and 10-year cause-specific survival in carcinoma group was carried out using univariate and Cox's multivariate analyses in which we chose 5years of age as a cut-point for staging of follicular carcinomas according to the new UICC stated by Dobert et al.,2004. Moreover, the tumor size in the statistical analyses was dichrotomized using the median $(35 \mathrm{~mm})$ as the cut-point. We found that the large tumors has a significantly poorer survival than the small ones. To improve the statistical power, the tumor size was tested in multivariate analysis as a continuous variable. Despite that, the tumor size as well as sex, nodal metastasis and cyclin D1 expression were not significant prognostic indicators while uni-and multivariate analyses showed that the age at time of diagnosis, distant metastasis, necrosis in primary tumor, the histologic degree of differentiation and extrathyroidal invasion as well as P53-immunodetection were significant prognostic factors for cause- specific survival because our statistical analyses revealed that the age over 45 years with poorly differentiated carcinomas, exhibiting tumor necrosis \& extrathyroid invasion (PT4) and showing distant metastasis together with P53 expression had unfavorable effect on survival. These data were simulating those mentioned by Goto et al.(2001); Lo et al.(2005) \& Siironen et al.(2005) who also stated that the expression of P53 in patients over 45 years of age could explain the more aggressive behavior of these tumors. In contrast, Hod et al.(2005) \& Besic et al.(2005) excluded the age as an independent prognostic factor for survival of thyroid follicular carcinomas.

\section{Conclusion}

Our study found that the age at time of diagnosis, the histologic differentiation, presence of distant metastases, necrosis in the primary tumor and extrathyroid 
invasion are important risk \& prognostic factors. However, the sex, the tumor size and presence of lymph nodal metastases are insignificant factors for survival. In addition, cyclin D1 detection may be involved in thyroid oncogenesis, and together with P53 expression, they may be incriminated in the progression of thyroid follicular neoplasms. Finally, expression of P53, but not cyclin D1, is an independent prognostic factor and may be valuable, with the above mentioned significant indicators, in the future planning of treatment of primary thyroid follicular carcinomas.

\section{References}

1. Battista S, Martelli MJ, Fedele M,Chiappetta G (1995): P53 in tumor differentiation. Oncogene., 11: 2029-37.

2. Besic N, Zgajnar J, Hocevar M, FrkovicGrazio S (2005): Is patient's age a prognostic factor for follicular thyroid carcinoma in the TNM classification system? Thyroid., 15 (5): 439-48.

3. Ceraline J, Deplangue $\mathbf{G}$, Noel $\mathbf{F}$, Natarajan-Ame $S$ (2003): Sensitivity to cisplatin treatment of human K1 thyroid carcinoma cell lines with altered P53 function. Cancer Chemother. Pharmacol., 51: 91-95.

4. Christian G, Pia A, Karsten J, Lars B, Per C (1998): Prognostic factors in papillary and follicular thyroid carcinomas: P53 expression is a significant indicator of prognosis. Laryngoscope, 108 (2): 243-9.

5. Dobashi Y, Sugimura H, Sakamoto A, Mernyei M (1994): Stepwise participation of P53 as a possible prognostic factor in human thyroid carcinoma. Am. J. Sur. Pathol., 17: 375-81.

6. Dobert $\mathbf{N}$, Menzel $\mathrm{C}$, Oeschger $\mathrm{S}$, Grunwald F (2004): Differentiated thyroid carcinoma: The new UICC 6th edition TNM classification system in a retrospective analysis of 169 patients. Thyroid., 14 (1): 65-70.

7. Fagin JA, Tang SH, Zeki K, Di Lauro R, Fusco A (1996): P53 in head and neck malignancies. Cancer Res., 56: 765-71.

8. Goto A, Sakamoto A, Machinami R (2001): An Immunohistochemical analysis of cyclin D1, P53 and P21 proteins in tumors originating from the thyroid gland. Pathol. Res. Pract., 197: 217-22.

9. Ho YS, Tseng SC, Chin TY, Hsieh LL (1996): P53 gene mutation in thyroid carcinoma. Cancer Letters., 103: 57-63.
10. Hod N, Hagag P, Baumer M, Sandbank J Horne T (2005): Differentiated thyroid carcinoma in children and young adult: Evaluation of response to treatment. J. Clin. Nucl. Med., 30 (6): 387-90.

11. Jensen RA and Page DL (1993): P53: The promissing story continues to unfold. Hum Pathol; 24: 455-6.

12. Josef MH (1999): Immunoreactivity of P53, Ki 67 and Bcl-2 in follicular adenomas and carcinomas of the thyroid gland. Hum. Pathol., 30 (8): 926-33.

13. Kataki A, Sotirianakos S, Memos N, Karayiannis M (2003): P53 and C-fos overexpression in patients with thyrtoid cancer. Neoplasma, 50 (1): 26-31.

14- Lazzeresci D, Sambuco L, Scalzo OC, Ranieri A (1998): Cyclin D1, Cyclin E and P53 expression in malignant thyroid cells in human thyroid carcinomas. Int. J. Cancer, 76: 1269-74.

15- Lo CY, Chan WF, Lam KY, Wan KY (2005): Follicular thyroid carcinoma: The role of histology and staging system in predicting survival. Ann. Surg, 242 (5): 708-15.

16- Marx J. (1993): Cell death studies yield cancer clue. Science, 259: 760-1.

17- Motoi N, Sakamoto A, Yamachi T, Horiuchi H (2000): Role of ras mutation in the progression of thyroid carcinoma of follicular epithelial origin. Pathol. Res. Pract., 196: 1-7.

18- Motokura T, Bloom T, Kim HG, Juppner H, Ruderman JV (1991): A novel cyclin encoded by a bcl-1-Linked candidate oncogene. Nature, 350: 512-5.

19- Nasir A,Catalano E,Calafati S, Cantor A (2004): Role of P53, CD 44 V6 and CD 57 in differentiating between benign and malignant follicular neoplasms of the thyroid. In Vivo, 18 ( 2) : 189-95.

20- Nishida N, Fukuda Y, Komeda T, Kita R (1994): Amplification and overexpression of the cyclin D1 gene in aggressive human hepatocellular carcinoma. Cancer Res., 54: 3107-10.

21- Okayasu I, Osakabe T, Onozawa M, Mikami T (1998): P53 and P21 expression in lymphocytic thyroiditis and thyroid tumors. J. Clin. Immunol. Immunopathol., 88 (2): 183-91.

22- Oltaviz AI and Korsmeyer SJ (1994): Checkpoints of duelling dimers foil death wishes. Cell, 79: 189-92.

23- Pilotti S, Collini P, Del Bo R, Pierotti MA (1994): A novel panel of antibodies that segregates immunocytochemically poorly differentiated carcinoma from 
undifferentiated carcinoma of thyroid gland. Am. J. Surg. Pathol., 18: 1054-64.

24- Plamero $I$ and Peters $S$ (1996): Perturbation of cell cycle regulators in human cancer. Cancer Surv., 27: 351-67.

25- Rosenberg CL, Wong E, Petty EM, Bale AE (1991): PRAD1, a candidate BCL-1 oncogene. Mapping and expression in centrocyte lymphoma. Proc. Natl. Acad. Sci. USA, 88: 9638-42.

26- Siironen P, Louhimo J, Nordling $\mathbf{S}$, Ristimaki A (2005): Prognostic factors in thyroid cancers: An evaluation of 601 consecutive patients. Tumor Biol., 26 (2): 57-64.

27- Soddu S, Blandino G, Scardigli R., Coen S (1996): P53 and Bcl-2 in colonic cancers. J. Cell. Biol., 134: 193-204.

28- Wang S, Lloyed RV, Hutzler MJ, Safran MS (2000): The role of cell cycle regulatory proteins, cyclin $\mathrm{D} 1$, in the progression of thyroid tumor. Med. Pathol., 13: 882-7.

29- Wang S, Wuu J,Savas L, Patwardharn NA, Khan A, (1998): The role of cell cycle regulatory proteins, cyclin D1, cyclin E, and P53 in thyroid carcinogenesis. Hum. Pathol., 29: 1304-9.

30- Wang XW and Harris CC (1996): The P53 tumor suppressor gene: Clues to molecular carcinogenesis and cancer therapy. Cancer Surv., 28: 169-96.

31- Watanabe M, Kuwano H, Tanaka S, Toh Y (1999): A significant morphological transformation is recognized in human oesophageal cancer cells with an amplification/overexpression of the cyclin D1 gene. Int. J. Oncol., 15: 1103-8.

32- Zhu XL, Hartwick W, Rohan T, Kandel R (1998): Cyclin D1 gene amplification and protein expression in benign breast disease and breast carcinoma. Mod. Pathol., 11: $1082-8$ 


\title{
إ ظهار مناعي هستو كيميائي للبروتينين Cyclin D1 , P

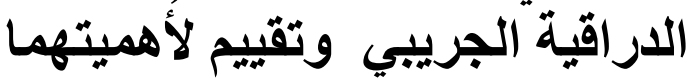

\author{
أحمد حسن عبد الرحمن الرشيدي (1) - سمير عبد السلام علي إبراهيم (2)ـعامر

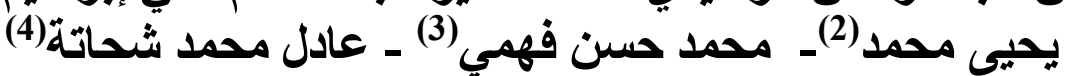

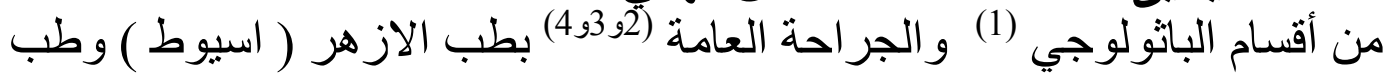 \\ القاهرة و المنيا.
}

لتوضيح أهمية البروتينين Cyclin D1 , P

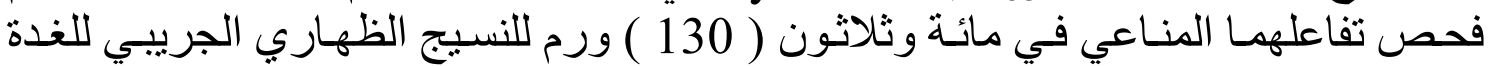

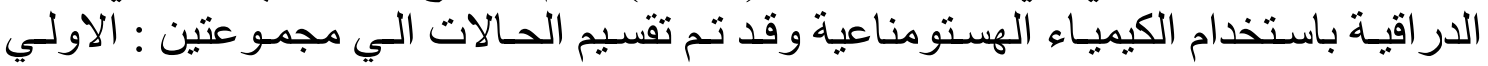

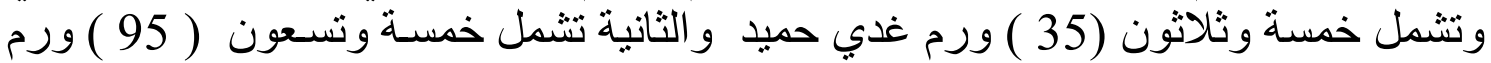

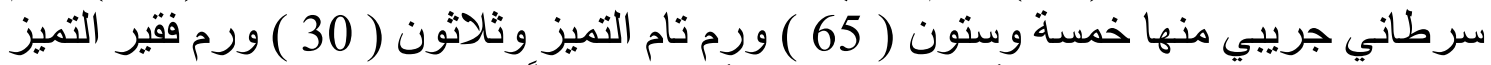

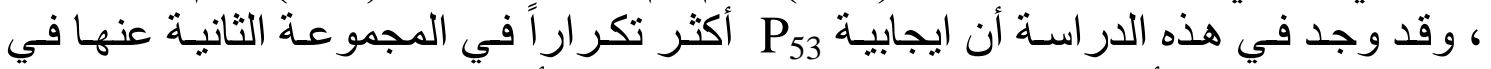

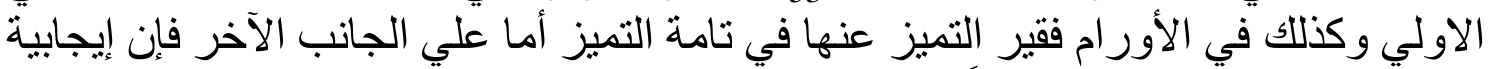

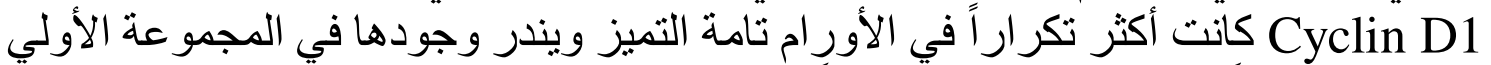

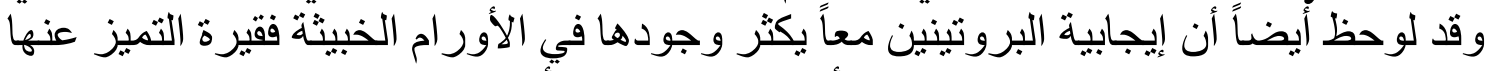

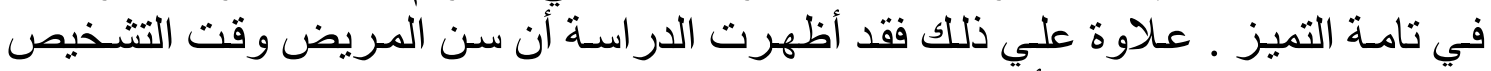

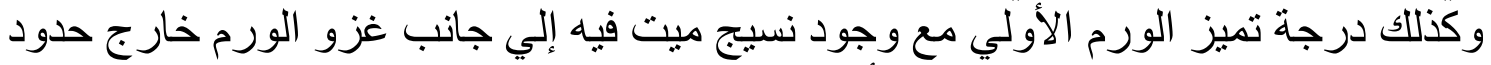

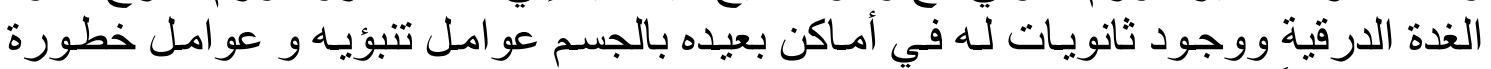

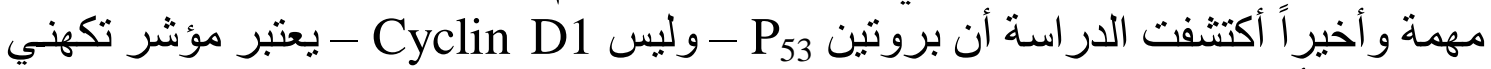

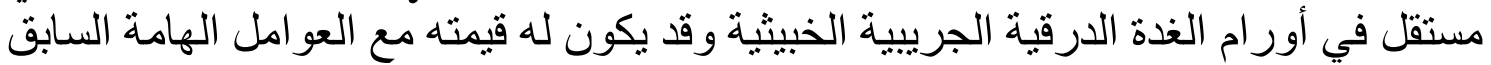

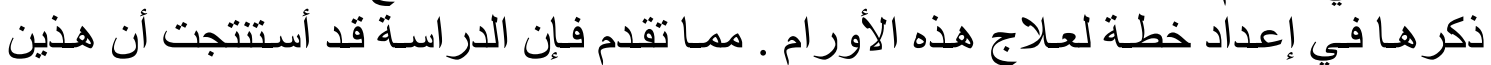

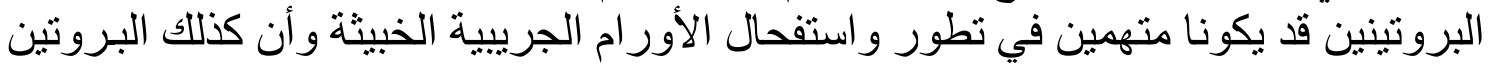
Cyclin D1 قد يشمله عمليه تكوين أورام الغدة الدرقية الجنين الجريبية ككل . 